PROCEEDINGS OF THE

AMERICAN MATHEMATICAL SOCIETY

Volume 127, Number 10, Pages 2981-2985

S 0002-9939(99)04879-0

Article electronically published on April 27, 1999

\title{
RANDOM FLUCTUATIONS OF CONVEX DOMAINS AND LATTICE POINTS
}

\author{
ALEX IOSEVICH AND KIMBERLY K. J. KINATEDER \\ (Communicated by Christopher D. Sogge)
}

\begin{abstract}
In this paper, we examine a random version of the lattice point problem. Let $\mathcal{H}$ denote the class of all homogeneous functions in $C^{2}\left(\mathbb{R}^{n}\right)$ of degree one, positive away from the origin. Let $\Phi$ be a random element of $\mathcal{H}$, defined on probability space $(\Omega, \mathcal{F}, P)$, and define

$$
\begin{aligned}
& \qquad F_{\Phi(\omega, \cdot)}(\xi)=\int_{\{x: \Phi(\omega, x) \leq 1\}} e^{-i\langle x, \xi\rangle} d x \\
& \text { for } \omega \in \Omega \text {. We prove that, if } E\left(\left|F_{\Phi}(\xi)\right|\right) \leq C[\xi]^{\frac{n+1}{2}} \text {, where }[\xi]=1+|\xi| \text {, then } \\
& \qquad E\left(N_{\Phi}\right)(t)=V t^{n}+O\left(t^{n-2+\frac{2}{n+1}}\right) \\
& \text { where } V=E(|\{x: \Phi(\cdot, x) \leq 1\}|) \text {, the expected volume. That is, on average, } \\
& N_{\Phi}(t)=V t^{n}+O\left(t^{n-2+\frac{2}{n+1}}\right) \text {. We give explicit examples in which the Gaussian } \\
& \text { curvature of }\{x: \Phi(\omega, x) \leq 1\} \text { is small with high probability, and the estimate } \\
& N_{\Phi}(t)=V t^{n}+O\left(t^{n-2+\frac{2}{n+1}}\right) \text { holds nevertheless. }
\end{aligned}
$$
\end{abstract}

\section{INTRODUCTION}

Let $\phi \in C^{\infty}\left(\mathbb{R}^{n} \backslash\{0\}\right)$ be homogeneous of degree one and positive in $\mathbb{R}^{n} \backslash\{0\}$. Let $N(t)=\#\left\{j \in \mathbb{Z}^{n}: \phi(j) \leq t\right\}, t>0$. It is well known (see e.g. [Rand69], [vdC21], [Hlw50], [So93]) that if $\Sigma_{\phi}=\{x: \phi(x)=1\}$ has everywhere non-vanishing Gaussian curvature, then

$$
N(t)=c t^{n}+O\left(t^{n-2+\frac{2}{n+1}}\right)
$$

In this paper we shall examine a random version of the lattice point problem. In [BCT96], the authors studied the expected number of lattice points inside randomly positioned convex polygons. The probability space in this case is the group of rotations and translations. We shall study the expected number of lattice points inside random convex domains, where the probability space can be more complicated. Our results are motivated by the following physical intuition. Consider a mass of dense elastic viscous material, expanding at roughly uniform rate. Suppose that at the time $t=1$, the boundary of the spill is convex. Suppose that the spill is subject to random fluctuations such as the air and water currents. It is not difficult to imagine that if the fluctuations are small, then the curvature of the boundary will remain non-negative. Let $N(t)$ denote the number of lattice points trapped by

Received by the editors September 17, 1997 and, in revised form, January 6, 1998.

1991 Mathematics Subject Classification. Primary 42Bxx; Secondary 60G99.

(C)1999 American Mathematical Society 
the spill at time $t$. The question we ask is, what conditions must be placed on these random fluctuation such that, on average, $N(t)$ behaves as in (1).

More precisely, we shall assume the following. Let $\mathcal{H}$ denote the class of all homogeneous functions in $C^{2}\left(\mathbb{R}^{n}\right)$ of degree one, positive away from the origin. Let $\Phi$ be a random element of $\mathcal{H}$. That is, given a probability space $(\Omega, \mathcal{F}, P)$ (where $\mathcal{F}$ is a $\sigma$-field on $\Omega$ and $P$ is a measure on $\Omega$ for which $P(\Omega)=1$ ), $\Phi$ is a measurable map from $\Omega$ to $\mathcal{H}$. Let

$$
F_{\Phi(\omega, \cdot)}(\xi)=\int_{\{x: \Phi(\omega, x) \leq 1\}} e^{-i\langle x, \xi\rangle} d x
$$

where $\omega \in \Omega$.

Let $E\left(\left|F_{\Phi}(\xi)\right|\right)$ denote the expected value of $\left|F_{\Phi(\omega, \cdot)}(\xi)\right|$; i.e. $E$ is integration with respect to probability measure $P$. Let

$$
N_{\Phi(\omega, \cdot)}(t)=\#\left\{j \in \mathbb{Z}^{n}: \Phi(\omega, j) \leq t\right\} .
$$

We shall see that if $E\left(\left|F_{\Phi}(\xi)\right|\right) \leq C[\xi]^{\frac{n+1}{2}}$, where $[\xi]=1+|\xi|$, then

$$
E\left(N_{\Phi}\right)(t)=V t^{n}+O\left(t^{n-2+\frac{2}{n+1}}\right)
$$

where $V=E(|\{x: \Phi(\cdot, x) \leq 1\}|)$, the expected volume. That is, on average, $N_{\Phi}(t)=V t^{n}+O\left(t^{n-2+\frac{2}{n+1}}\right)$.

The point here is the following. As we mentioned above, in order to the get the estimate (1), it suffices to have the non-vanishing Gaussian curvature of the level set $\{\phi=1\}$. This leads to the favorable estimate of the Fourier transform of the indicator function of the set $\{\phi \leq 1\}$, which results in the estimate (1). We shall exhibit random elements $\Phi$ of $\mathcal{H}$ (see Theorem 2 below), for which a number of realizations $\Phi(\omega, \cdot)$ results in $\{x: \Phi(\omega, x)=1\}$ having subsets of arbitrarily small Gaussian curvature. Nevertheless, the expected value of the Gaussian curvature at each point of $\{x: \Phi(\cdot, x)=1\}$ remains positive, and so the expected number of lattice points will be as in (1).

\section{STATEMENT OF RESULTS}

Theorem 1. Let $\Phi$ be a random element of $\mathcal{H}$. Suppose that $E\left(\left|F_{\Phi}(\xi)\right|\right) \leq C[\xi]^{-\frac{n+1}{2}}$. Then the estimate (4) holds.

The proof of Theorem 1 is an easy modification of the classical deterministic case. See, for example, [So93], Ch.1.

Remark. It is known (see e.g [Rand69]) that if the probability space is $S O_{n}$, then the assumptions of Theorem 1 are satisfied. It is also known (see e.g. [BCT96]) that if the probability space is $S O_{n}$ together with the translation group, then the error estimate in (4) is actually $O\left(t^{\frac{n-1}{2}}\right)$.

The better error estimate, $O\left(t^{\frac{n-1}{2}}\right)$, is the result of averaging over the rotation group instead of the more general situation considered in Theorem 1 and the examples below. However, at least in the dimension $n=2$, the estimate $O\left(t^{\frac{n-1}{2}+\epsilon}\right)=O\left(t^{\frac{1}{2}+\epsilon}\right), \epsilon>0$, is conjectured to be the right result in the deterministic situation in the case of the circle. The best current result in this direction is due to Huxley who obtained $O\left(t^{\frac{43}{76}}\right)$. (See [Hux97].)

In the following theorem we give non-trivial examples of random $\Phi \in \mathcal{H}$ and accompanying probability spaces $(\Omega, \mathcal{F}, P)$ such that the estimate $\left|F_{\Phi(\omega, \cdot)}(\xi)\right| \leq$ 
$C(\omega)[\xi]^{-\frac{n+1}{2}}$ has the unfortunate property that $C(\omega)$ is arbitrarily large. However, the estimate (4) still holds.

Before stating the theorem, we will give a brief introduction to Brownian motion. Readers familiar with this topic may skip to the statement of Theorem 2.

Recall that a probability set $(\Omega, \mathcal{F}, P)$ consists of a set $\Omega$ together with $\sigma$-field $\mathcal{F}$ and measure $P$ on $\Omega$ such that $P(\Omega)=1$.

We begin by defining a random variable on probability space $(\Omega, \mathcal{F}, P)$ to be a real-valued measurable map on $\Omega$. A Gaussian random variable $X$ with mean $\mu$ and standard deviation $\sigma$ is one where

$$
P(X \in A)=\int_{A} \frac{1}{\sqrt{2 \pi \sigma^{2}}} e^{-(x-\mu)^{2} / 2 \sigma^{2}} d x
$$

for all Borel sets $A$ in $\mathbb{R}$.

Now define $\mathcal{B}$ to be the Borel sigma-field on $[0, \infty)$. A stochastic process $X_{t}(\omega)$ (or just $X_{t}$ ) is a map from $[0, \infty) \times \Omega$ to $\mathbb{R}$ which is measurable with respect to the product sigma-field of $\mathcal{B}$ and $\mathcal{F}$.

Definition 1. A stochastic process $X_{t}$ is said to be a one-dimensional Brownian motion starting at 0 if the following conditions are satisfied:

(i) $P\left(X_{0}=0\right)=1$;

(ii) for all $s \leq t, X_{t}-X_{s}$ is a Gaussian random variable with mean 0 and variance $t-s$;

(iii) for all $s<t, X_{t}-X_{s}$ is independent of $\sigma\left(X_{u}, u \leq s\right)$, where $\sigma\left(X_{u}, u \leq s\right)$ denotes the smallest sigma-field with respect to which each $X_{u}, u \leq s$, is measurable;

(iv) $P\left(X_{t}\right.$ is continuous in $\left.t\right)=1$.

Several proofs are available for the existence of this stochastic process. See, for example, [Dur91] or any measure-theoretic introduction to probability theory. It is of interest to mention that for all $\omega$ outside a set of probability 0 , Brownian motion $X .(\omega)$ is nowhere differentiable.

We will be concerned, however, with Brownian motion starting at some point other than 0. To consider Brownian motion starting at some $x \in \mathbb{R}$, we can either just consider the process $x+X_{t}$ or utilize the following more useful approach.

Definition 2. Let $X_{t}$ be Brownian motion as defined in Definition 1. Let $\Omega$ be the set of continuous functions $\omega(\cdot)$ from $[0, \infty)$ to $\mathbb{R}$, without the requirement that $\omega(0)=0$. Let $W_{t}$ be defined on $\Omega$ such that

$$
W_{t}(\omega)=\omega(t),
$$

and let $\mathcal{F}$ be the smallest sigma-field with respect to which each $W$. is measurable. Next, define the probability measure $P_{x}$ on this $(\Omega, \mathcal{F})$ by

$$
P_{x}(W . \in A)=P(x+X . \in A),
$$

for all $x \in \mathbb{R}$ and $A \in \mathcal{F}$.

It is by this convention that we shall specify the starting point of Brownian motion in the first two parts of the theorem that follows.

Theorem 2. Let $\left\{W_{s}\right\}_{s>0}$ denote real-valued Brownian motion as defined in Definition 2, together with measures $\left\{P_{x}\right\}$. Let $T$ denote the first hitting time of 1 ; that is, $T=\inf \left\{s>0: W_{s}=1\right\}$. Define $X_{s}=W_{\min \{s, T\}}$, and let $Y_{s}$ solve the equation $Y_{s}^{\prime \prime}=\frac{1}{X_{s}}, 0<s \leq 1, Y_{0}=Y_{1}=1$. Let $\Gamma=\left\{Y_{s} e^{2 \pi i s}: s \in[0,1]\right\}$. Let $\Phi_{X}$ 
be the random element of $\mathcal{H}$ such that $\Gamma(\omega)=\Sigma_{\Phi_{X}(\omega, \cdot)}=\left\{\Phi_{X}(\omega, \cdot)=1\right\}, \forall \omega \in \Omega$. Let $K(\omega, \cdot)$ denote the Gaussian curvature of $\Sigma_{\Phi(\omega, \cdot)}$. Then $P_{2}\{|K(s)|<\epsilon\}>0$, $\forall \epsilon>0$. However, the estimate (4) holds.

Again, let $\left\{W_{s}\right\}_{s>0}$ denote real-valued Brownian motion as defined in Definition 2, together with measures $\left\{P_{x}\right\}$. Let $Z_{s}=W_{s} \chi_{\left\{W_{s} \geq 2\right\}}+\left(e^{W_{s}-2}+1\right) \chi_{\left\{W_{s}<2\right\}}$ and define $\Phi_{Z}$ and $K$ as above with $X$ replaced by $Z$. Then $P_{2}\{|K(s)|<\epsilon\}>0, \forall \epsilon>0$. However, the estimate (4) holds.

Fix $n \in \mathbb{N}$. On an arbitrary probability space $(\bar{\Omega}, \overline{\mathcal{F}}, \bar{P})$, let $S_{k}^{j}, j=1, \ldots, n$, denote independent random walks, where $S_{k}^{j}=\sum_{i=1}^{k} \xi_{i}^{j}$, for $\xi_{i}^{j}$ independent and identically distributed random variables on $\bar{\Omega}$ such that $\bar{P}\left(\xi_{i}^{j}=1\right)=\bar{P}\left(\xi_{i}^{j}=-1\right)=$ $\frac{1}{2}$, for all $i, j$. Let $T^{j}=\inf \left\{i>1:\left|S_{i}^{j}\right|=3\right\}$. Define the stochastic process $U_{s}$ as the linear interpolation of points $\left(\frac{j}{n}, T^{j}\right)$, and let $Y_{s}^{\prime \prime}=\frac{1}{U_{s}}, 0<s \leq 1$. Let $\Phi_{U}$ and $K$ be defined as above with $X$ replaced by $U$. Then $\bar{P}(|K(s)|<\epsilon)>0$. However, the estimate (4) holds.

Remark. We can think of the domain bounded by $\Sigma_{\Phi_{X}}$ as a mass of elastic but viscous material undergoing random fluctuations. In the first example, $\Phi_{X}$ describes a mass where a segment of the boundary is undergoing random fluctuations. This is illustrated by the fact that, with probability one, a segment of the boundary $\Sigma_{\Phi_{X}}$ of random length does not fluctuate much, since $Y_{s}^{\prime \prime}$ is constant there.

We think of the domains bounded by $\Sigma_{\Phi_{Z}}$ and $\Sigma_{\Phi_{U}}$ as masses of more elastic materials, since the curvature function is very wild.

Note that the processes $X, Z$ and $U$ are of the following basic type. Let $X_{t}$ : $\Omega \times[0,1] \rightarrow(\epsilon, \infty)$. We just need that for all $M, P\left(X_{t}>M\right.$ for some $\left.t\right)>0$. We then solve $Y^{\prime \prime}=\frac{1}{X}$ and the desired result follows.

\section{ProOF OF THEOREM 2}

To simplify notation, we shall write $E$ for $E_{2}$ and for $\bar{E}$ (expectation with respect to $\bar{P})$, taken in context.

To prove that (4) holds in the above examples, it is enough to show that

$$
E\left(\sup \left|X_{s}\right|\right)<\infty \text { and } E\left(\sup \left|Z_{s}\right|\right)<\infty \text { and } E\left(\sup \left|U_{s}\right|\right)<\infty
$$

since the assumptions of Theorem 1 are satisfied if the expected value of the Gaussian curvature is bounded from below. Recall (see e.g [So93]) that if the Gaussian curvature of the boundary of a set is bounded from below, the Fourier transform of the indicator function of a set decays of order $-\frac{n+1}{2}$.

We first deal with $\Phi_{X}$. It is not hard to see that

$$
E\left(\left(\inf Y_{s}^{\prime \prime}\right)^{-1}\right)=E\left(\sup X_{s}\right) \leq E\left(\sup _{s<1} W_{s}\right) \text {. }
$$

Using the fact that $E\left(\sup _{s<u} W_{s}\right)=E\left(\left|W_{u}\right|\right)$, for $u>0$ (see, for example, [KS91], pg. 96), we take $u=1$ and compute

$$
E\left(\left(\inf Y_{s}^{\prime \prime}\right)^{-1}\right) \leq \int_{-\infty}^{\infty}|x|(2 \pi u)^{\frac{-1}{2}} \exp \left(\frac{-x^{2}}{2 u}\right) d x=\sqrt{\frac{2 u}{\pi}}<\infty .
$$

In order to see that

$$
P\{|K(s)|<\epsilon\}>0 \quad \forall \epsilon>0,
$$


recall that $Y_{s}^{\prime \prime}=\frac{1}{X_{s}}$. Moreover,

$$
P\left\{\left|\frac{1}{X(s)}\right|<\delta\right\}=P\left\{\left|W_{\min \{s, T\}}\right|>\frac{1}{\delta}\right\} \geq P\left\{\left|W_{s}\right|>\frac{1}{\delta}\right\}>0 \quad \forall \delta>0,
$$

by elementary properties of the normal distribution.

We now deal with $\Phi_{Z}$. It is not hard to see that

$$
E\left(\sup \left|Z_{s}\right|\right) \leq E\left(2+\sup \left|W_{s}\right|\right) \leq 2+E\left(\left|W_{1}\right|\right)=2+\sqrt{\frac{2}{\pi}}<\infty,
$$

as computed in (10).

In order to see that

$$
P\{|K(s)|<\epsilon\}>0 \quad \forall \epsilon>0,
$$

recall that $Y_{s}^{\prime \prime}=\frac{1}{Z_{s}}$. Hence, it is enough to take $\delta<\frac{1}{2}$ without loss of generality and use again that $W_{s}$ is a normal random variable, so that

$$
P\left\{\frac{1}{Z_{s}}<\delta\right\}=P\left\{W_{s}>\frac{1}{\delta}\right\}>0 .
$$

To finish the proof, we consider now $\Phi_{U}$.

$$
E\left(\left(\inf Y_{s}^{\prime \prime}\right)^{-1}\right)=E\left(\sup X_{s}\right)=E\left(\sup _{1 \leq j \leq n} T^{j}\right) .
$$

Since $E\left(T^{j}\right)<\infty$ and $\sup _{1 \leq j \leq n} T^{j}$ is the $n^{t h}$ order statistic of the $T^{j}$ 's, we can see that $E\left(\sup _{1<j<n} T^{j}\right)<\infty$ and, in particular, that $E\left(\sup _{1<j<n} T^{j}\right)<n E\left(T^{1}\right)$.

Finally, $P\{|K(s)|<\epsilon\}>0 \quad \forall \epsilon>0$ follows from

$$
P\left\{\frac{1}{U_{s}}<\delta\right\}=P\left\{\left|T^{j}\right|>\frac{1}{\delta} \text { for some } j\right\}>0,
$$

by properties of the random walk.

This completes the proof of Theorem 2.

\section{REFERENCES}

[vdC21] J. G. van der Corput, Zahlentheoretische Abschatzungen, Math. Ann. 84 (1921).

[Dur91] R. Durrett, Probability: Theory and Examples, Wadsworth and Brooks-Cole (1991). MR 91m:60002

[Hlw50] E. Hlawka, Uber Integrale auf konvexen Korper I, Monatsh Math 54 (1950).

[Hux97] M. Huxley, Area, Lattice Points, and Exponential Sums, London Math. Soc. Mon. New Series 13 (1996). MR 97g:11088

[KS91] I. Karatzas and S. E. Shreve, Brownian Motion and Stochastic Calculus, Springer Verlag (1991). MR 92h:60127

[Rand69] B. Randol, On the asymptotic behavior of the Fourier transform of the indicator function of a convex set, Trans. of the A.M.S. 139 (1969), 279-285. MR 40:4678b

[So93] C. D. Sogge, Fourier Integrals in Classical Analysis, Cambridge Tracts in Math \#105 (1993). MR 94c:35178

[BCT96] L. Brandolini, L. Colzani, and G. Travaglini, Average decay of the Fourier transform, and lattice points inside polyhedra, (preprint) (1996).

Department of Mathematics, Georgetown University, Washington, DC 20057

E-mail address: iosevich@math.georgetown.edu

Department of Mathematics and Statistics, Wright State University, Dayton, Ohio 45435

E-mail address: kjk@euler.math.wright.edu 